\title{
Infarct Patterns in the Middle Cerebral Artery Territory and Their Association with Large Artery Stenosis
}

\author{
Martin Osorio Flores ${ }^{1}$, Y Swamy Venkatesh ${ }^{2 *},{\text { Ambika } \text { Rao }^{3} \text { and Michael Brooks }}^{4}$ \\ ${ }^{1}$ Department of Neurology, Drexel University College of Medicine, Philadelphia, Pennsylvania, USA \\ ${ }^{2}$ Department of Neurology, University of South Carolina School of Medicine, Philadelphia, Pennsylvania, USA \\ ${ }^{3}$ Department of Medicine, Dorn VAMC, Columbia, SC, Philadelphia, Pennsylvania, USA \\ ${ }^{4}$ Department of Neuroradiology, Mercy Catholic Medical Center, Philadelphia, Pennsylvania, USA
}

"Corresponding author: Y Swamy Venkatesh, Department of Neurology, University of South Carolina School of Medicine, 8 Medical Park, Suite 420, Columbia, SC 29203, USA, Tel: 803-545-6050; Fax: 803-545-6051; E-mail: yvenkatesh@uscmed.sc.edu

Rec date: Mar 18, 2014; Acc date: Apr 29, 2014; Pub date: May 03, 2014

Copyright: (c) 2014 Osorio-Flores M, et al. This is an open-access article distributed under the terms of the Creative Commons Attribution License, which permits unrestricted use, distribution, and reproduction in any medium, provided the original author and source are credited.

\begin{abstract}
Background and Purpose: We investigated the association between critical stenosis of the internal carotid artery or the first segment of the middle cerebral artery and patterns of cerebral infarcts classified by their territorial distribution.

Methods: This cross-sectional study included patients with diffusion-weighted magnetic resonance-confirmed acute/early subacute infarcts in the middle cerebral artery territory who underwent head/neck magnetic resonance angiography. We grouped infarct patterns in two categories: shower/cortical watershed/superficial perforating territory and complete middle cerebral artery/cortical territorial/deep perforating territory.
\end{abstract}

Results: Seventeen and 33 patients, respectively, presented with shower/cortical watershed/superficial perforating territory infarcts and complete middle cerebral artery/cortical territorial/deep perforating territory infarcts. Eleven $(64.7 \%)$ of the former and five $(15.2 \%)$ of the latter had critical stenosis of the internal carotid artery or the first segment of the middle cerebral artery. A strong statistical association favoring the presence of critical stenosis in the shower/cortical watershed/superficial perforating territory group $(P=.001)$ persisted on logistic regression analysis $(P=.002)$.

Conclusions: The occurrence of diffusion-weighted magnetic resonance-confirmed acute/early subacute middle cerebral artery infarcts in the shower/cortical watershed/superficial perforating territory distribution is strongly associated with the presence of critical stenosis of the internal carotid artery or the first segment of the middle cerebral artery. Further prospective studies are needed to address the significance of this finding.

\section{Introduction}

The relationship between different distribution patterns of cerebral infarcts and the presence of internal carotid artery (ICA) or middle cerebral artery (MCA) stenosis has been studied with inconsistent results [1-17]. Classification of cerebral infarcts affecting the cortical territories supplied by the three major cerebral arteries has been largely accepted, although anatomical studies have demonstrated significant variability [18]. Classification of subcortical infarcts is inherently more difficult and prone to dissimilarities. Most studies distinguish between infarcts in the area of the perforating arteries (the terminal arteries leaving the larger cortical vessels and penetrating the white matter into deeper levels) and "internal borderzone" infarcts, considered to be located at the border of two facing, perforating vascular systems. However, this distinction is not consistently defined in the literature, with infarcts in the same territory, particularly in the deepest areas of the brain, classified within the perforating territory (particularly the superficial perforating territory) in some studies and within the internal borderzone territory in others.

We sought to determine the presence of an association between critical stenosis of either the ICA or the first segment (M1) of the
MCA and different patterns of cerebral infarcts classified according to their territorial distribution. In summary, the occurrence of acute/ early subacute middle cerebral artery infarcts in the shower/cortical watershed/superficial perforating territory distribution is strongly associated with the presence of critical stenosis of the internal carotid artery or the first segment of the middle cerebral artery.

\section{Methods}

This was a retrospective, cross-sectional study. Approval was received from the Mercy Catholic Medical Center institutional review board. We included patients who presented with new-onset neurological symptoms to a community-based hospital, with diffusion-weighted magnetic resonance imaging (MRI)-confirmed acute or early subacute infarcts in the territory of the MCA who also underwent diagnostic MR angiography (MRA) evaluation of the head and neck arteries. We considered MCA territory to be the maximum MCA territory as described in the anatomical study by van der Zwan et al. [18]. To assess carotid or MCA stenosis we used time-of-flight MRA. Images were obtained in a 1.5-T machine within 7 days of admission. All the images were read by the study neuroradiologist 
Page 2 of 4

(MB), who was not aware of the classification of the infarct patterns described below, as this was designed only before the retrospective data review.

We grouped infarct patterns in two categories :1) a group comprised of cortical-predominant multifocal, "shower" infarcts (S), cortical watershed infarcts (W), and superficial perforating territory infarcts (Ps), hereafter called SWPs and 2) a group formed by complete MCA territory infarcts $(\mathrm{M})$, cortical-predominant territorial infarcts $(\mathrm{T})$, and deep perforating territory infarcts $(\mathrm{Pd})$, which we called MTPd. The classification of infarcts into the territories of the superficial and deep perforating arteries followed the templates from Bogousslavsky and Regli [19] and Ghika et al. [20]. We did not use the term "internal borderzone" infarcts, as this name is used to describe infarcts that ultimately have the same distribution and postulated pathophysiology as superficial perforating territory infarcts when different papers mentioning one or the other term are compared $[2,21]$.

The group division used herein was made prior to the data review, considering previous studies and empirical knowledge associating the infarct patterns in the first group (as opposed to those in the second group) with the presence of large artery stenosis. Although there were very few cases presenting with more than one infarct pattern belonging to the same group, there were no cases showing infarct patterns of both groups, which supported the appropriateness of the classification [2-4,21].

We also obtained information regarding demographic characteristics of the patients at presentation and the presence of other cardiovascular risk factors such as hypertension, diabetes mellitus, hypercholesterolemia, smoking, atrial fibrillation, recent myocardial infarction, or prosthetic heart valve. In our study, hypertension was defined as the use of antihypertensive medications on admission plus a history of hypertension, or a systolic blood pressure of $140 \mathrm{mmHg}$ or greater, or a diastolic blood pressure of $90 \mathrm{~mm} \mathrm{Hg}$ or greater on admission. Diabetes mellitus was defined as the use of hypoglycemic drugs on admission or an HbA1c level greater than $6.5 \%$ at any time during the hospitalization. Hypercholesterolemia was defined as the use of statins or ezetimibe on admission or total cholesterol of 200 $\mathrm{mg} / \mathrm{dL}$ or greater during hospitalization. Smoking was defined as the use of any number of cigarettes within 28 days prior to admission. Atrial fibrillation was defined as a history or new diagnosis of paroxysmal or persistent atrial fibrillation. Recent myocardial infarction was defined as a history of myocardial infarction within 3 months prior to admission or myocardial infarction developing during the hospitalization. Prosthetic heart valve was defined as the presence of a mechanical or bioprosthetic heart valve replacement.

The primary objective of this study was to compare the presence of critical stenosis of either the ipsilateral ICA or the M1 segment of the ipsilateral MCA between the two study groups (SWPs vs. MTPd). The degree of stenosis on MRA was measured according to the North American Symptomatic Carotid Endarterectomy Trial (NASCET) criteria [22]. It was defined as the remaining lumen at the stenosis as percentage of the normal lumen distal to the stenosis. Critical stenosis was defined as either high-grade stenosis (70\%-99\%) or occlusion (100\%).

For statistical analysis we used SPSS 18.0 software (IBM Corp., Somers, NY). For categorical variables we used the $\chi^{2}$ test and for continuous variables (age) we used the independent-samples $t$ test. For all categorical dichotomous variables we used a continuity correction for the $\chi^{2}$ value. We considered a $P$ value of less than 0.05 as statistically significant. We performed a logistic regression analysis to adjust the results for possible confounding variables.

\section{Results}

There were a total of 278 admissions to the hospital between January 2008 and December 2009 with a main discharge diagnosis of cerebral infarct. Of those, 114 patients were excluded for not having a diffusion-weighted MRI; 86 for not having an MRA either of the head or neck; 17 for having an infarct not classified as belonging to the MCA territory; and 11 for having a diffusion-weighted MRI negative for acute/early subacute infarct. Fifty patients met the inclusion criteria for the study. Medical records and imaging studies were analyzed as previously described.

\begin{tabular}{|l|l|l|l|}
\hline & $\begin{array}{l}\text { MTPd } \\
\mathrm{n}=33\end{array}$ & $\begin{array}{l}\text { SWPs } \\
\mathrm{n}=17\end{array}$ & $P$ value \\
\hline Mean age (SD) & $66.64(13.4)$ & $66.59(11.6)$ & .990 \\
\hline Male sex & $17(51.5 \%)$ & $11(64.7 \%)$ & .556 \\
\hline Hypertension & $22(66.7 \%)$ & $12(70.6 \%)$ & 1.000 \\
\hline Diabetes mellitus & $10(30.3 \%)$ & $6(35.3 \%)$ & .969 \\
\hline Hypercholesterolemia & $18(54.5 \%)$ & $11(37.9 \%)$ & .699 \\
\hline Smoking & $7(21.2 \%)$ & $8(47.1 \%)$ & .118 \\
\hline Atrial fibrillation & $5(15.2 \%)$ & $1(5.9 \%)$ & .620 \\
\hline ICA/MCA critical stenosis & $5(15.2 \%)$ & $11(64.7 \%)$ & .001 \\
\hline
\end{tabular}

Table 1: Demographic Features, Cardiovascular Risk Factors, and Large Artery Stenosis in Patients with MTPd and SWPs Infarct Patterns

A total of 17 patients presented with an infarct pattern within the SWPs category whereas 33 patients showed an infarct of the MTPd category. There were no statistically significant differences in the mean age or male-to-female ratio between the two study groups (Table 1). Similarly, there were no differences in the frequency of hypertension, diabetes mellitus, hypercholesterolemia, atrial fibrillation, or smoking 
Page 3 of 4

between the two study groups. There were no cases of recent myocardial infarction or prosthetic heart valve in either of the two groups.

Of the 17 cases included in the SWPs group, 11 (64.7\%) had critical stenosis of the ipsilateral ICA or the M1 segment of the ipsilateral MCA whereas six had no evidence of it. Of the 33 cases within the MTPd group, five (15.2\%) had and 28 (84.8\%) did not have ipsilateral critical stenosis of either of these arteries. Even though the MTPd group had more patients in this study only a minority of patients in this subgroup had ipsilateral stenosis of either of these arteries in contrast to the SWPs group.
Comparing the presence of either ipsilateral ICA or M1 critical stenosis between the two study groups showed a strong statistical significance towards a higher frequency of critical stenosis in the group composed of cortical-predominant multifocal, cortical watershed, and superficial perforating territory infarcts $(P=.001)$. This difference persisted on the logistic regression analysis even after we adjusted for age, sex, hypertension, diabetes mellitus, hypercholesterolemia, atrial fibrillation, and smoking $(P=.002$; Table 2).

\begin{tabular}{|l|l|l|l|}
\hline & $P$ Value & Odds Ratio & $95 \% \mathrm{Cl}$ \\
\hline Mean age & .550 & 1.02 & $.95-1.10$ \\
\hline Male sex & .533 & 1.68 & $.33-8.59$ \\
\hline Hypertension & .378 & 0.39 & $.05-3.12$ \\
\hline Diabetes mellitus & .579 & 1.89 & $.19-18.10$ \\
\hline Hypercholesterolemia & .935 & 1.08 & $.18-6.64$ \\
\hline Smoking & .098 & 5.38 & $.74-39.39$ \\
\hline Atrial fibrillation & .620 & 0.16 & $.01-2.17$ \\
\hline ICA/MCA critical stenosis & .002 & 20.09 & $3.12-124.43$ \\
\hline
\end{tabular}

Table 2: Logistic Regression Analysis of Demographic Features, Cardiovascular Risk Factors, and Large Artery Stenosis Comparing SWPs with MTPd Infarct Patterns

\section{Discussion}

The results of this study indicate that the occurrence of diffusionweighted MRI-confirmed acute/early subacute MCA-territory cerebral infarcts in the cortical-predominant multifocal (shower), cortical watershed, or superficial perforating distribution, when these infarcts are taken as a group, is strongly associated with the presence of critical ipsilateral ICA or M1 stenosis as assessed by MRA. This association persists after adjusting for other important cardiovascular risk factors and atrial fibrillation.

Studies evaluating the association of infarct patterns with large artery stenosis have had variable results. Different systems of classification of subcortical infarcts may account for the dissimilar results. In this study we made an effort not to use ambiguous definitions and eliminated the term "internal borderzone" because when different papers are compared, this name is used to describe infarcts that have the same distribution and postulated pathophysiology as superficial perforating territory infarcts.

We found no statistically significant difference in the frequency of atrial fibrillation between the two groups and there were no cases of recent myocardial infarction or prosthetic heart valve. Although these were the only three criteria for cardioembolismfrom the TOAST classification of acute stroke (Trial of Org 10172 in Acute Stroke Treatment) [23] used here, our main goal was not to look for the primary cause of cerebral infarcts but to identify the presence of a more immediate association with large artery stenosis, which is the finding that most frequently leads to planning for interventional treatment.
We used MR angiography as the only vascular imaging method in this study. A large meta-analysis investigating the accuracy of MRA for the detection of ICA stenosis (with most of the studies using NASCET criteria) showed that the sensitivity of time-of-flight MRA for the detection of $70 \%-99 \%$ stenosis was $91.2 \%$ and specificity was $88.3 \%$. The sensitivity of MRA for the detection of ICA occlusion was $94.5 \%$ and its specificity was $99.3 \%$ [24]. A study assessing the accuracy of 1.5-T time-of-flight MRA for the detection of intracranial artery disease, with M1 segment disease being included along with other intracranial arterial segments, found that the sensitivity and specificity for stenosis greater than $49 \%$ were $95 \%$ and $96 \%$, respectively. The sensitivity and specificity to detect occlusion were both $100 \%$ as compared with digital subtraction angiography [25]. Therefore, although MRA is not the gold standard for the assessment of large artery stenosis, its widespread use, convenience, and fewer contraindications compared with conventional angiography, along with an acceptable sensitivity and specificity, support the use of this diagnostic modality in larger association studies.

Limitations of our study include its retrospective and crosssectional nature and its relatively small sample size. With the aim of obtaining objective evidence of acute/early subacute infarcts and large artery stenoses and making our study comparable with others evaluating this association, we did not include patients with no diffusion-weighted MRI or MRA, which might have led to selection bias in any direction. It should also be noted that patterns of cerebral infarcts may not only be dependent on the causative pathophysiology but also on the pre-existence of collaterals and on the integrity of the circle of Willis. 
The association we found between the proposed groups of infarct patterns and critical ICA and M1 stenosis meets statistical significance and merits further investigation with prospective studies using the anatomically based classification designed in this study. In terms of its practical application, even though it is true that no infarct pattern should preclude the performance of vascular studies, particularly in the community setting, not every patient undergoes this work-up and therefore, objective decision-making criteria would at least increase the yield of detection of a potentially modifiable risk factor.

\section{Acknowledgments}

The authors would like to thank Dr. Edward Gracely (Department of Family, Community, and Preventive Medicine, Drexel University College of Medicine) for his valuable help with the statistical analysis of the study and Dr. Rashmi Balasubramanya (Department of Radiology, Mercy Catholic Medical Center) for her help in obtaining the digital images.

\section{References}

1. Bogousslavsky J, Regli F (1986) Borderzone infarctions distal to internal carotid artery occlusion: prognostic implications. Ann Neurol 20: 346-350.

2. Bladin CF, Chambers BR (1993) Clinical features, pathogenesis, and computed tomographic characteristics of internal watershed infarction. Stroke 24: 1925-1932.

3. Lee PH, Oh SH, Bang OY, Joo SY, Joo IS, et al. (2004) Infarct patterns in atherosclerotic middle cerebral artery versus internal carotid artery disease. Neurology 62: 1291-1296.

4. Gandolfo C, Del Sette M, Finocchi C, Calautti C, Loeb C (1998) Internal borderzone infarction in patients with ischemic stroke. Cerebrovasc Dis 8: 255-258.

5. Yong SW, Bang OY, Lee PH, Li WY (2006) Internal and cortical borderzone infarction: clinical and diffusion-weighted imaging features. Stroke 37: 841-846.

6. Lee PH, Oh SH, Bang OY, Joo IS, Huh K (2004) Isolated middle cerebral artery disease: clinical and neuroradiological features depending on the pathogenesis. J Neurol Neurosurg Psychiatry 75: 727-732.

7. Angeloni U, Bozzao L, Fantozzi L, Bastianello S, Kushner M, et al. (1990) Internal borderzone infarction following acute middle cerebral artery occlusion. Neurology 40: 1196-1198.

8. Kang DW, Chu K, Ko SB, Kwon SJ, Yoon BW, et al. (2002) Lesion patterns and mechanism of ischemia in internal carotid artery disease: a diffusion-weighted imaging study. Arch Neurol 59: 1577-1582.

9. Nakano S, Yokogami K, Ohta H, Goya T, Wakisaka S (1995) CT-defined large subcortical infarcts: correlation of location with site of cerebrovascular occlusive disease. AJNR Am J Neuroradiol 16: 1581-1585.

10. Wong KS, Gao S, Chan YL, Hansberg T, Lam WW, et al. (2002) Mechanisms of acute cerebral infarctions in patients with middle cerebral artery stenosis: a diffusion-weighted imaging and microemboli monitoring study. Ann Neurol 52: 74-81.
11. Hupperts RM, Warlow CP, Slattery J, Rothwell PM (1997) Severe stenosis of the internal carotid artery is not associated with borderzone infarcts in patients randomised in the European Carotid Surgery Trial. J Neurol 244: 45-50.

12. Lee PH, Oh SH, Bang OY, Joo IS, Huh K (2005) Pathogenesis of deep white matter medullary infarcts: a diffusion weighted magnetic resonance imaging study. J Neurol Neurosurg Psychiatry 76: 1659-1663.

13. Yonemura K, Kimura K, Minematsu K, Uchino M, Yamaguchi T (2002) Small centrum ovale infarcts on diffusion-weighted magnetic resonance imaging. Stroke 33: 1541-1544.

14. Tsiskaridze A, Devuyst G, de Freitas GR, van Melle G, Bogousslavsky J (2001) Stroke with internal carotid artery stenosis. Arch Neurol 58: 605-609.

15. Lee PH, Bang OY, Oh SH, Joo IS, Huh K (2003) Subcortical white matter infarcts: comparison of superficial perforating artery and internal borderzone infarcts using diffusion-weighted magnetic resonance imaging. Stroke 34: 2630-2635.

16. Del Sette M, Eliasziw M, Streifler JY, Hachinski VC, Fox AJ, et al. (2000) Internal borderzone infarction: a marker for severe stenosis in patients with symptomatic internal carotid artery disease. For the North American Symptomatic Carotid Endarterectomy (NASCET) Group. Stroke 31: 631-636.

17. Read SJ, Pettigrew L, Schimmel L, Levi CR, Bladin CF, et al. (1998) White matter medullary infarcts: acute subcortical infarction in the centrum ovale. Cerebrovasc Dis 8: 289-295.

18. van der Zwan A, Hillen B, Tulleken CA, Dujovny M, Dragovic L (1992) Variability of the territories of the major cerebral arteries. J Neurosurg 77: 927-940.

19. Bogousslavsky J, Regli F (1992) Centrum ovale infarcts: subcortical infarction in the superficial territory of the middle cerebral artery. Neurology 42: 1992-1998.

20. Ghika JA, Bogousslavsky J, Regli F (1990) Deep perforators from the carotid system. Template of the vascular territories. Arch Neurol 47: 1097-1100.

21. Zülch KJ (1985) The Cerebral Infarct: Pathology, Pathogenesis, and Computed Tomography. Berlin: Springer Verlag.

22. North American Symptomatic Carotid Endarterectomy Trial Collaborators (1991) Beneficial effect of carotid endarterectomy in symptomatic patients with high-grade carotid stenosis. N Engl J Med 325: 445-453.

23. Adams HP Jr, Bendixen BH, Kappelle LJ, Biller J, Love BB, et al. (1993) Classification of subtype of acute ischemic stroke. Definitions for use in a multicenter clinical trial. TOAST. Trial of Org 10172 in Acute Stroke Treatment. Stroke 24: 35-41.

24. Debrey SM, Yu H, Lynch JK, Lövblad KO, Wright VL, et al. (2008) Diagnostic accuracy of magnetic resonance angiography for internal carotid artery disease: a systematic review and meta-analysis. Stroke 39: 2237-2248.

25. Sadikin C, Teng MM, Chen TY, Luo CB, Chang FC, et al. (2007) The current role of $1.5 \mathrm{~T}$ non-contrast $3 \mathrm{D}$ time-of-flight magnetic resonance angiography to detect intracranial steno-occlusive disease. J Formos Med Assoc 106: 691-699. 